\title{
DISAGREEMENT, CORRELATION AND ASSET PRICES
}

\author{
XUE-ZHONG HE AND LEI SHI \\ Finance Discipline Group \\ University of Technology, Sydney \\ PO Box 123 Broadway \\ NSW 2007, Australia
}

\begin{abstract}
When people agree to disagree, how does the disagreement affect asset prices? Within an equilibrium framework with two agents, two risky assets and a riskless bond, we analyze the joint impact of disagreement about expected payoff, variance and correlation, and compare prices with benchmark prices in a market with homogeneous beliefs.
\end{abstract}

JEL Classification: G12, D84.

Keywords: equilibrium asset prices, disagreement, consensus belief, mispricing, spillover effect.

\section{INTRODUCTION}

In financial markets, it is well recognized that people agree to disagree and the disagreement can have a significant impact on asset prices (see for example Fama and French (2007)). Disagreement complicates the formulation of asset prices, which makes a complete analysis difficult. In a static setting, when investors with the same risk tolerance agree on the covariance matrix, several authors have shown that assets remain correctly priced and the disagreement effect "cancels out" when beliefs about expected returns are heterogeneous but on average unbiased (see for example Levy, Levy and Benita (2006) and Yan (2010)). The analysis becomes much more complicated when there is a disagreement about the covariance matrix, because investors' demands are non-linear functions of their beliefs of the covariance matrix. Recently, Chiarella, Dieci and He (2011) show that, when asset payoffs are uncorrelated, disagreement about variances leads to a diversification effect. However, Duchin and Levy (2010) show that tiny fluctuations in the disagreement about the variance lead to substantial price fluctuations. Moreover, most of the literature focuses on the price impact of a specific type of disagreement (expected returns or variances) by assuming investors are otherwise identical, and not much attention has been paid to their joint impact, which can be very different from their individual

Date: March 5, 2012.

We would like to thank a referee for the helpful comments and valuable suggestions. The usual caveat applies. Corresponding author: Lei Shi, Finance Discipline Group, University of Technology, Sydney, PO Box 123 Broadway, NSW 2007, Australia. Email: Lei.Shi@uts.edu.au. Ph: (61 2) 9514 1972. Fax: (61 2) 95147711. 
impact. For example, Jouini and Napp (2006, 2008) and Chiarella et al. (2011) find that the impact of disagreement on prices is governed by the risk tolerance weighted average level of pessimism/optimism.

In a market with two risky assets, agents may have different risk tolerances, and jointly disagree about the expected payoffs, variances of payoffs and the correlation between payoffs. We show that even when agents have the same objective belief about the expected payoff and variance for the first asset, the market as a whole can be overoptimistic/overpessimistic and overconfident about its payoff if agents simultaneously disagree about the expected payoff and variance of the second asset or simultaneously disagree about the expected payoff of the second asset and the correlation between payoffs. As a result, prices of both assets are in general different from the benchmark prices in a market with homogeneous beliefs. This leads to a spillover effect of disagreement in a multi-asset market. All our results are limited to a static model. Impact of disagreement in a dynamic model can be very different. For example, Jouini and Napp (2011) show that even when beliefs are on average unbiased and risk tolerances are the same, disagreement can have a significant impact on the price dynamics and the risk-return trade-off of risky assets.

This paper is organized as follows, Section 2 presents an equilibrium asset pricing model with heterogeneous beliefs, Section 3 analyzes the impact of disagreement on asset prices and Section 4 concludes.

\section{THE MODEL}

We consider a two-date economy with two risky assets, indexed by $k=1,2$, a riskless bond, and two agents, indexed by $i=1,2$. The bond is in zero net supply and each agent is endowed with one share of each risky asset on date zero. The future payoff of asset $k$ is denoted by $X_{k}$ and let $\mathbf{X}=\left(X_{1}, X_{2}\right)^{T}$, moreover, the risk-free rate is assumed to be zero and the current price of the bond is 1 . The asset payoffs are assumed to be jointly normal and agents are assumed to have heterogeneous beliefs about the expected payoffs and covariance matrix of the payoffs. For agent $i(i=1,2)$, let

$$
\boldsymbol{\mu}_{i} \equiv\left(\mu_{i, 1}, \mu_{i, 2}\right)^{T}, \quad V_{i} \equiv\left(\begin{array}{cc}
\sigma_{i, 1}^{2} & \rho_{i} \sigma_{i, 1} \sigma_{i, 2} \\
\rho_{i} \sigma_{i, 1} \sigma_{i, 2} & \sigma_{i, 2}^{2}
\end{array}\right),
$$

where $\mu_{i, k}=\mathbb{E}_{i}\left(X_{k}\right), \sigma_{i, k}^{2}=\operatorname{Var}_{i}\left(X_{k}\right), \quad \rho_{i}=\operatorname{Correl}_{i}\left(X_{1}, X_{2}\right)$ for $i, k=1,2$, and denote $\mathcal{B}_{i}:=\left(\boldsymbol{\mu}_{i}, V_{i}\right)$ the subjective belief of agent $i$.

2.1. Portfolio Optimization. The terminal wealth of agent $i$ is given by $W_{i}=z_{i, B}+\mathbf{z}_{i}^{T} \mathbf{X}$, where $\mathbf{z}_{i}=\left(z_{i, 1}, z_{i, 2}\right)^{T}$ is the number of shares of the risky assets held by agent $i$, and $z_{i, B}$ is the number of bonds held. Agent $i$ maximizes a constant absolute risk aversion (CARA) utility function $U_{i}\left(W_{i}\right)=-\tau_{i} \exp \left\{-W_{i} / \tau_{i}\right\}$ of his terminal wealth $W_{i}$ under his subjective belief $\mathcal{B}_{i}$, 
subject to the budget constraint $\mathbf{z}_{i}^{T} \mathbf{p}+z_{i, B}=\mathbf{p}^{T} \mathbf{1}$, where $\tau_{i}$ is agent $i$ 's risk-tolerance. When the terminal wealth $W_{i}$ is normally distributed, maximizing $\mathbb{E}_{i}\left[U_{i}\left(W_{i}\right)\right]$ is equivalent to maximizing the certainty equivalent wealth given by $z_{i, B}+\mathbf{z}_{i}^{T} \boldsymbol{\mu}_{i}-\frac{1}{2 \tau_{i}} \mathbf{z}_{i}^{T} V_{i} \mathbf{z}_{i}$, where $\mathbf{p}=\left(p_{1}, p_{2}\right)^{T}$ is the equilibrium price vector of the risky assets. Therefore, the optimal portfolio of agent $i$ is given by

$$
\mathbf{z}_{i}^{*}=\tau_{i} V_{i}^{-1}\left(\boldsymbol{\mu}_{i}-\mathbf{p}\right) \quad \text { and } \quad z_{i, B}^{*}=\mathbf{p}^{T}\left(\mathbf{1}-\mathbf{z}_{i}^{*}\right)
$$

2.2. Consensus Belief and Market Equilibrium. The market clearing conditions are given by $\frac{1}{2}\left(\mathbf{z}_{1}^{*}+\mathbf{z}_{2}^{*}\right)=\mathbf{1}$ and $z_{1, B}+z_{2, B}=0$. Note that agents' budget constraints imply that

$$
\mathbf{p}^{T} \mathbf{1}=\frac{1}{2}\left(\mathbf{z}_{1}^{*}+\mathbf{z}_{2}^{*}\right)^{T} \mathbf{p}+\frac{1}{2}\left(z_{1, B}+z_{2, B}\right) .
$$

Therefore, the bond market clears as long as the asset market clears.

To characterize market equilibrium under heterogeneous beliefs, a concept of consensus belief has been developed by Lintner (1969) and Rubinstein (1974, 1975). In this paper, a belief $\mathcal{B}_{a}=\left(\boldsymbol{\mu}_{a}, V_{a}\right)$ is called a market consensus belief if the equilibrium prices under the heterogeneous beliefs $\mathcal{B}_{i}:=\left(\boldsymbol{\mu}_{i}, V_{i}\right)(i=1,2)$ are also the equilibrium prices under the homogeneous belief $\mathcal{B}_{a}$.

We construct a consensus belief similar to Chiarella et al. (2011), which allows us to analyze the heterogeneous economy as an equivalent homogeneous economy. Let $\tau_{a}=\frac{1}{2}\left(\tau_{1}+\tau_{2}\right)$ be the average risk tolerance. Applying Proposition 3.2 in Chiarella et al. (2011), the consensus belief $\mathcal{B}_{a}$ is given by

$$
V_{a}^{-1}=\frac{1}{2}\left[\frac{\tau_{1}}{\tau_{a}} V_{1}^{-1}+\frac{\tau_{2}}{\tau_{a}} V_{2}^{-1}\right], \quad \boldsymbol{\mu}_{a}=\frac{1}{2}\left[\frac{\tau_{1}}{\tau_{a}}\left(V_{a} V_{1}^{-1}\right) \boldsymbol{\mu}_{1}+\frac{\tau_{2}}{\tau_{a}}\left(V_{a} V_{2}^{-1}\right) \boldsymbol{\mu}_{2}\right] ;
$$

and the equilibrium asset prices are given by

$$
\mathbf{p}=\boldsymbol{\mu}_{a}-V_{a} \mathbf{1} / \tau_{a}
$$

Furthermore, the equilibrium optimal portfolio of agent $i$ is given by

$$
\mathbf{z}_{i}^{*}=\tau_{i} V_{i}^{-1}\left[\left(\boldsymbol{\mu}_{i}-\boldsymbol{\mu}_{a}\right)+V_{a} \mathbf{1} / \tau_{a}\right] .
$$

In the following, we use the consensus belief constructed in equation (3) to examine the impact of disagreement among agents on the equilibrium prices (4) of risky assets.

\section{The Price Impact of Disagreements}

To measure the price impact of disagreement, we first consider a benchmark economy in which agents have homogeneous beliefs and the same level of risk tolerance, that is, $\mathcal{B}_{i}=$ $\mathcal{B}_{o}=\left(\boldsymbol{\mu}_{o}, V_{o}\right)$, where $\mathcal{B}_{o}$ may be regarded as the objective belief about the distribution of asset payoffs and $\tau_{i}=\tau$. Since there is no disagreement, the consensus belief in this case coincides 
with the objective belief, that is

$$
\boldsymbol{\mu}_{a}=\boldsymbol{\mu}_{o} \equiv\left(\mu_{1}, \mu_{2}\right)^{T}, \quad V_{a}=V_{o} \equiv\left(\begin{array}{cc}
\sigma_{1}^{2} & \rho \sigma_{1} \sigma_{2} \\
\rho \sigma_{1} \sigma_{2} & \sigma_{2}^{2}
\end{array}\right)
$$

and the equilibrium asset prices under homogeneous belief, or the benchmark prices, denoted by $\hat{\mathbf{p}}$ are given by

$$
\hat{\mathbf{p}}=\left(\mu_{1}-\left(\sigma_{1}^{2}+\rho \sigma_{1} \sigma_{2}\right) / \tau, \mu_{2}-\left(\sigma_{2}^{2}+\rho \sigma_{1} \sigma_{2}\right) / \tau\right)^{T}
$$

For the economy with heterogeneous beliefs, we assume that agents agree about the expected payoff and standard deviation of the first asset $\left(S_{1}\right)$, that is, $\left(\sigma_{i, 1}, \mu_{i, 1}\right)=\left(\sigma_{1}, \mu_{1}\right)$ for $i=1,2$. Furthermore, there is a disagreement about the expected payoff and standard deviation of the second asset $\left(S_{2}\right)$, and also the correlation between asset payoffs. The disagreement among agents is measured by

$$
\Delta \mu \equiv \mu_{1,2}-\mu_{2,2}, \Delta \sigma \equiv \sigma_{1,2}-\sigma_{2,2}, \text { and } \Delta \rho \equiv \rho_{1}-\rho_{2} .
$$

When $\Delta \mu>(<) 0$, agent 1 is relatively more optimistic (pessimistic) about the payoff of $S_{2}$ than agent 2 ; when $\Delta \sigma>(<) 0$, agent 1 is relatively more doubtful (confident) about the payoff of $S_{2}$ than agent 2 ; when $\Delta \rho>(<) 0$, agent 1 perceives a higher (lower) correlation between asset payoffs than agent 2. Moreover, assume the average risk tolerance is given by $\tau_{a}=\tau$, the difference in risk tolerance is measured by $\Delta \tau \equiv \tau_{1}-\tau_{2}$. Hence, when $\Delta \tau>(<) 0$, agent 1 is more (less) risk tolerant than agent 2 . Following (4), the equilibrium prices are then determined by the consensus belief,

$$
\mathbf{p}=\left(\mu_{a, 1}-\left(\sigma_{a, 1}^{2}+\rho_{a} \sigma_{a, 1} \sigma_{a, 2}\right) / \tau, \mu_{a, 2}-\left(\sigma_{a, 2}^{2}+\rho_{a} \sigma_{a, 1} \sigma_{a, 2}\right) / \tau\right)^{T} .
$$

If consensus belief coincides with the objective belief, then $\mathbf{p}=\hat{\mathbf{p}}$.

To facilitate the analysis, we introduce notations of three different averages, namely the arithmetic, geometric and harmonic averages, defined by

$$
A\left(x_{1}, x_{2}\right) \equiv\left(x_{1}+x_{2}\right) / 2, \quad G\left(x_{1}, x_{2}\right) \equiv \sqrt{x_{1} x_{2}}, \quad H\left(x_{1}, x_{2}\right) \equiv\left[\left(1 / x_{1}+1 / x_{2}\right) / 2\right]^{-1} .
$$

Note that, when $x_{1} \neq x_{2}$, we have $H\left(x_{1}, x_{2}\right)<G\left(x_{1}, x_{2}\right)<A\left(x_{1}, x_{2}\right)$. To examine the impact of the disagreement, we consider three cases.

Case 1. The impact of risk tolerance and optimism/pessimism - This case has been considered in the literature. For example, in a market with a single risky asset, Jouini and Napp (2007) show that the consensus belief of the expected payoff is a risk-tolerance weighted average of agents' perceived expected payoffs. We show in the next proposition 1 that this result also carries over to a multi-asset market.

\footnotetext{
${ }^{1}$ Proofs of propositions only involve simple algebras, therefore are omitted from the paper.
} 
Proposition 1. When $\Delta \sigma=\Delta \rho=0$, the consensus belief is given by $V_{a}=V_{o}, \boldsymbol{\mu}_{a}=$ $\left(\mu_{1}, \alpha \mu_{1,2}+(1-\alpha) \mu_{2,2}\right)^{T}$, where $\alpha \equiv \tau_{1} /\left(\tau_{1}+\tau_{2}\right)$. The equilibrium prices are given by

$$
p_{1}=\hat{p}_{1}, \quad p_{2}=\hat{p}_{2}+\alpha\left(\mu_{1,2}-\mu_{2}\right)+(1-\alpha)\left(\mu_{2,2}-\mu_{2}\right) .
$$

Proposition 1 shows that $S_{1}$ is always correctly priced in the sense that its price coincides with the benchmark price of $S_{1}$, which is intuitive since both agents perceive the objective expected payoff for $S_{1}$ and the objective covariance matrix. For $S_{2}$, the consensus belief is a risk-tolerance weighted average of agents' perceived expected payoffs, which is also intuitive since the more risk tolerant agent would buy/sell $S_{2}$ more aggressively than the less risk tolerant agent. This result is consistent with Jouini and Napp (2006, 2007) and Chiarella et al. (2011).

Case 2. The impact of optimism/pessimism and confidence/doubt - In this case, agents have the same risk tolerances and perceive the same expected payoff and variance for $S_{1}$ and the same correlation between asset payoffs, but disagree about the expected payoff and standard deviation of $S_{2}$.

Proposition 2. When $\Delta \tau=0$ and $\Delta \rho=0$, the consensus belief $\mathcal{B}_{a}$ is given by

$$
\mu_{a, 1}=\mu_{1}-\frac{1}{2} \frac{\rho \sigma_{1}}{\phi} \Delta \sigma \Delta \mu, \quad \mu_{a, 2}=\beta \mu_{1,2}+(1-\beta) \mu_{2,2}
$$

and

$$
\rho_{a}=\gamma_{\rho} \rho, \quad \sigma_{a, 1}^{2}=\gamma \sigma_{1}^{2}, \quad \sigma_{a, 2}^{2}=\left[\omega A\left(\frac{1}{\sigma_{1,2}^{2}}, \frac{1}{\sigma_{2,2}^{2}}\right)+(1-\omega) G\left(\frac{1}{\sigma_{1,2}^{2}}, \frac{1}{\sigma_{2,2}^{2}}\right)\right]^{-1},
$$

where

$$
\phi \equiv\left(2-\rho^{2}\right) A\left(\sigma_{1,2}^{2}, \sigma_{2,2}^{2}\right)-\rho^{2} G\left(\sigma_{1,2}^{2}, \sigma_{2,2}^{2}\right)>0, \quad \beta \equiv \frac{\left(2-\rho^{2}\right) \sigma_{2,2}^{2}-\rho^{2} \sigma_{1,2} \sigma_{2,2}}{2 \phi},
$$

and

$$
\gamma_{\rho} \equiv \frac{A\left(\sigma_{1,2}, \sigma_{2,2}\right)}{\sqrt{A\left(\sigma_{1,2}^{2}, \sigma_{2,2}^{2}\right)}}<1, \quad \gamma \equiv \frac{1-\rho^{2}}{1-\rho^{2} \frac{A\left(\sigma_{1,2}, \sigma_{2,2}\right)^{2}}{A\left(\sigma_{1,2}^{2}, \sigma_{2,2}^{2}\right)}} \leq 1, \quad \omega \equiv \frac{2-\rho^{2}}{2\left(1-\rho^{2}\right)} \geq 1
$$

Proposition 2 shows that disagreement can lead to a spillover effect when asset payoffs are correlated, that is when $\rho$ different from zero. Although agents perceive the objective expected payoff for $S_{1}$, equation (7) implies that the market (represented by the consensus belief) can be overoptimistic/overpessimistic about $S_{1}$ 's payoff. For example, if $\rho>(<) 0$, a positive correlation between confidence and optimism about $S_{2}$ 's payoff, that is $\Delta \sigma \Delta \mu<0$, can lead to overoptimism (overpessimism) about the payoff $\left(\mu_{a, 1}>(<) \mu_{1}\right)$ of $S_{1}$, which contributes to overpricing (underpricing) of $S_{1}$. Furthermore, equation (8) implies that, although agents perceive the same correlation coefficient and the same variance for $S_{1}$, the market is overconfident about $S_{1}$ 's payoff $\left(\sigma_{a, 1}^{2}<\sigma_{1}^{2}\right)$ and perceives a lower correlation between asset payoffs 
$\left(\rho_{a}<\rho\right)$, both of which contribute to overpricing of $S_{1}$ when the correlation coefficient $\rho>0$. In summary, when asset payoffs are correlated and agents disagree about the expected payoff and variance of $S_{2}$, even though agents perceives the objective expected payoff and variance for $S_{1}$ and the objective correlation, the market can be overoptimistic/overpessimistic and overconfident about $S_{1}$ 's payoff and also perceives a lower correlation. The spillover effect disappears when $\rho=0$, in this case, market perceives the objective expected payoff and variance for $S_{1}$ and the objective correlation, that is, $\mu_{a, 1}=\mu_{1}, \sigma_{a, 1}^{2}=\sigma_{1}^{2}, \rho_{a}=\rho=0$, and $p_{1}=\hat{p}_{1}=\mu_{1}-\sigma_{1}^{2} / \tau$.

Equation (8) shows that the consensus belief about $S_{2}$ 's precision $\left(1 / \sigma_{a, 2}^{2}\right)$ is a weighted average of the arithmetic and geometric means of agents' perceived precisions, and the weight on the geometric mean is negative since $\omega>1$. When $\rho=0$, we obtain $\omega=1$ and the consensus belief about $S_{2}$ 's variance becomes a harmonic mean of agents' perceived variances of $S_{2}$, that is $\sigma_{a, 2}^{2}=H\left(\sigma_{1,2}^{2}, \sigma_{2,2}^{2}\right)$. When $\rho$ is different from zero, because $G\left(\frac{1}{\sigma_{1,2}^{2}}, \frac{1}{\sigma_{2,2}^{2}}\right)<A\left(\frac{1}{\sigma_{1,2}^{2}}, \frac{1}{\sigma_{2,2}^{2}}\right)$, market perceives a higher precision (hence a lower variance) for $S_{2}$ compare to the case of $\rho=0$. Furthermore, equation (7) shows that the consensus belief about $S_{2}$ 's expected payoff is a weighted arithmetic average of agents' perceived expected payoffs and the weights are determined by the objective correlation and the perceived variances for $S_{2}$. When $\rho=0$, the consensus belief about $S_{2}$ 's expected payoff becomes a precision-weighted average of the perceived expected payoffs, that is $\beta=\frac{1 / \sigma_{1,2}^{2}}{1 / \sigma_{1,2}^{2}+1 / \sigma_{2,2}^{2}}$, which is consistent with Jouini and Napp (2007).

Case 3: Optimism/Pessimism and disagreement in correlations - In this case, we examine the joint impact of optimism/pessimism about $S_{2}$ (measured by $\Delta \mu$ ) and disagreement in the correlation coefficient (measured by $\Delta \rho$ ) by assuming $\Delta \tau=0, \Delta \sigma=0$. To our knowledge, this is the first paper to examine the impact of disagreement about correlation on asset prices.

Proposition 3. When $\Delta \tau=\Delta \sigma=0$, the consensus belief $\mathcal{B}_{a}$ is given by

$$
\mu_{a, 1}=\mu_{1}-\frac{1}{4} \frac{\sigma_{1}}{\sigma_{2}} \frac{\Delta \rho \Delta \mu}{\phi_{\rho}}, \quad \mu_{a, 2}=\theta \mu_{1,2}+(1-\theta) \mu_{2,2}
$$

and

$$
\rho_{a}=\omega_{\rho} \rho_{1}+\left(1-\omega_{\rho}\right) \rho_{2}, \quad \sigma_{a, 1}^{2}=\gamma_{1} \sigma_{1}^{2}, \quad \sigma_{a, 2}^{2}=\gamma_{2} \sigma_{2}^{2},
$$

where

$$
\phi_{\rho}=1-A\left(\rho_{1}, \rho_{2}\right)^{2}>0, \quad \theta=\frac{1-\rho_{2} A\left(\rho_{1}, \rho_{2}\right)}{2 \phi_{\rho}}>0
$$

and

$$
\omega_{\rho} \equiv\left[1+\frac{1-\rho_{1}^{2}}{1-\rho_{2}^{2}}\right]^{-1}, \quad \gamma_{1}=\gamma_{2}=\frac{1-A\left(\rho_{1}^{2}, \rho_{2}^{2}\right)}{1-A\left(\rho_{1}, \rho_{2}\right)^{2}}<1
$$

In Proposition 3, equation (9) shows that even though agents perceive the same expected payoff for $S_{1}$, the market is overpessimistic (overoptimistic) about the $S_{1}$ 's payoff when there is 
a positive (negative) correlation between optimism and perceived correlations, that is $\Delta \rho \Delta \mu>$ $(<) 0$, which contributes to underpricing (overpricing) of $S_{1}$. Furthermore, the consensus belief about $S_{2}$ 's payoff $\mu_{a, 2}$ is a weighted average of agents perceived expected payoffs, weights are determined by agents' perceived correlations. When the arithmetic average of the perceived correlations is zero, that is $A\left(\rho_{1}, \rho_{2}\right)=0$, we obtain $\theta=\frac{1}{2}$ and $\mu_{a, 2}=A\left(\mu_{1}, \mu_{2}\right)$.

Moreover, equation (10) shows that even though agents perceive the same variances for the payoffs of both assets, market is overconfident about the asset payoffs, which contributes to the overpricing of assets when $\sigma_{a, 1}>-\rho_{a} \sigma_{a, 2}$ and $\sigma_{a, 2}>-\rho_{a} \sigma_{a, 1}$. The consensus belief about the correlation is a weighted average of agents' perceived correlations and the weights are biased towards the agent who perceives a higher absolute correlation, $\left|\rho_{i}\right|$. When $A\left(\rho_{1}, \rho_{2}\right)=0$, agents perceive the same absolute correlation $\left(\left|\rho_{1}\right|=\left|\rho_{2}\right|\right)$ and market perceives the average perceived correlation, that is $\rho_{a}=A\left(\rho_{1}, \rho_{2}\right)=0$.

\section{CONCLUSION}

In a market with two risky assets and a riskless bond, we show that disagreement about the expected payoff and variance of one asset, together with disagreement about the correlation coefficient, can jointly affect the consensus belief about the payoffs of both assets, leading to a spillover effect. Prices determined by the consensus belief are in general different from the benchmark prices in a market with homogeneous beliefs. However, our results are limited to the static model and an extension to a dynamic model would be interesting, which we leave to future research.

\section{REFERENCES}

Chiarella, C., Dieci, R. and He, X. (2011), 'Do heterogeneous beliefs diversify market risk?' European Journal of Finance 17, 241-258.

Duchin, R. and Levy, M. (2010), 'Disagreement, portfolio optimization and excess volatility', Journal of Financial and Quantitative Analysis 45, 623-640.

Fama, E. and French, K. (2007), 'Disagreement, tastes, and asset prices', Journal of Financial Economics 83, 667689.

Jouini, E. and Napp, C. (2006), 'Heterogeneous beliefs and asset pricing in discrete time: An analysis of pessimism and doubt', Journal of Economic Dynamics and Control 30, 1233-1260.

Jouini, E. and Napp, C. (2007), Strategic beliefs. SSRN Working Paper, Abstract ID 950508.

Jouini, E. and Napp, C. (2008), 'Are more risk averse agents more optimistic? Insights from a rational expectations model', Economics Letters 101, 73-76.

Jouini, E. and Napp, C. (2011), 'Unbiased disagreement in financial markets, waves of pessimism and the riskreturn trade-off', Review of Finance 15, 575-601.

Levy, M., Levy, H. and Benita, G. (2006), 'Capital asset prices with heterogeneous beliefs', Journal of Business 79, 1317-1353.

${ }^{2}$ The condition is always satisfied when $\rho_{a} \geq 0$. 
Lintner, J. (1969), ‘The aggregation of investor's diverse judgements and preferences in purely competitive security markets', Journal of Financial and Quantitative Analysis 4, 347-400.

Rubinstein, M. (1974), 'An aggregation theorem for securities markets', Journal of Financial Economics 1, 225244.

Rubinstein, M. (1975), 'Security market efficiency in an arrow-debreu economy', American Economic Review 65, 812-824.

Yan, H. (2010), 'Is noise trading cancelled out by aggregation?' Management Science 57, 1047-1059. 